\title{
Erratum to: Neutron reflectometry by refractive encoding
}

R. Cubitt ${ }^{1, a}$ and J. Stahn ${ }^{2}$

1 Institut Laue Langevin (ILL) - 6 Jules Horowitz, 38000 Grenoble, France

2 Paul Scherrer Institut, Laboratory for Neutron Scattering WHGA/142 - CH-5232, Villigen PSI, Switzerland

Original article: Eur. Phys. J. Plus 126: 111, DOI: 10.1140/epjp/i2011-11111-0

Received: 5 February 2016

Published online: 29 March 2016 - (c) Società Italiana di Fisica / Springer-Verlag 2016

The first part of eq. (1) should have been

$$
\varphi=\cos ^{-1}(n \cos (\alpha))-\alpha,
$$

and eq. (5) should have been

$$
\Delta \lambda=(\mathrm{d} \varphi / \mathrm{d} \lambda)^{-1}\left[\left(\left(s_{1}+2\right) /\left(2 D_{1}\right)\right)^{2}+\Delta f^{2}+\left(((\varphi+\alpha) L+\Delta x) /\left(2 D_{2}\right)\right)^{2}\right]^{1 / 2} .
$$

\footnotetext{
${ }^{a}$ e-mail: cubitt@ill.fr
} 